\title{
Demonstration of Strong Forward-Backward Asymmetry in the C1s Photoelectron Angular Distribution from Oriented CO Molecules
}

\author{
F. Heiser, O. Geßner, J. Viefhaus, K. Wieliczek, R. Hentges, and U. Becker \\ Fritz-Haber-Institut der Max-Planck-Gesellschaft, D-14195 Berlin, Germany
}

(Received 19 March 1997)

\begin{abstract}
Angular distributions of $\mathrm{C}(1 s)$ photoelectrons emitted from oriented $\mathrm{CO}$ molecules were measured using a new method: mass and angle-resolved photoelectron-photoion coincidence spectroscopy. Along with the angular momentum composition of the photoelectron wave, the experiment reveals pronounced forward-backward asymmetries in the photoemission intensity with respect to the molecular orientation. This asymmetry, being observed for the first time in molecular photoionization, is interpreted in terms of multiple scattering effects. Calculations using the MS-X $\alpha$ method show good quantitative agreement with the presented experimental results. [S0031-9007(97)04163-X]
\end{abstract}

PACS numbers: 33.80. .Eh

Molecular photoionization differs from atomic photoionization in two distinct ways: one is the possibility of fragmentation of the ionized system and the other one is the occurrence of intramolecular scattering of photoelectrons and possibly also Auger electrons on the different atomic constituents of the molecule. It is the latter which gives rise to such prominent features as shape resonances in molecular $K$-shell photoionization [1]. Intramolecular scattering, however, is not only exhibited in the variation of the partial photoionization cross section of valence or $K$-shell photoelectrons, it is also the crucial process determining the angular momentum composition and, consequently, the energy-dependent behavior of the emitted photoelectrons, including interference effects. Dehmer and Dill $[1,2]$ were the first who predicted that $f$-wave enhancement in the $\sigma$ channel was the source of the photoelectron intensity enhancement observed in molecular shape resonances. It took almost twenty years before this prediction could be verified experimentally in a direct way.

Shigemasa et al. [3] showed in a pioneering experiment on $\mathrm{N}_{2}$ molecules fixed in space that the angular momentum of the outgoing photoelectron in the vicinity of the shape resonance was indeed predominantly of $f$-wave character. Their experiment opened the door to angular momentum resolved photoelectron spectroscopy in molecular photoionization. In a series of subsequent experiments the same group studied similar behavior in other molecules including heteronuclear diatomic and triatomic molecules [4,5]. They all showed in their shape resonances some $f$-wave enhancement as seen in $\mathrm{N}_{2}$ but with less intense pronunciation. In addition to this angular momentum specific behavior a subsequent paper of Dill, Siegel, and Dehmer [6] predicted another phenomenon characteristic of molecular photoionization: strong forward-backward asymmetries or site specific angular distributions of photoelectrons emitted from heteronuclear molecules fixed in space. This effect, which has been predicted by a theoretical method describing the problem in terms of multiple electron scattering on the different sites of the atomic constituents of a molecule, has so far never been proven experimentally.

In this Letter we report on the first experimental evidence of asymmetric photoemission in molecular photoionization, an effect occurring due to cylindrical symmetry breaking and interference between the outgoing partial photoelectron waves. The experimental results will be compared with the twenty-year-old prediction by the MS-X $\alpha$ method of Dill, Siegel, and Dehmer [6] showing unprecedented agreement with our measurements.

The experiments were performed at beam line BW3 of HASYLAB [7,8]. The key element of this experiment is a time-of-flight ion spectrometer with a positionsensitive anode optimized for short dead time [9]. This ion detector makes it possible to trace the momentum direction of the ions at the instant of photoionization. Such measurement is a direct probe of the orientation of the molecule when the photoelectron is emitted if one assumes that the dissociation time is fast compared to the molecular rotation: a condition known as "axial recoil approximation" [10].

Figure 1 shows a scheme of the experimental setup used for this angular correlation experiment between ionic fragments and photoelectrons. A weak repelling field for ions was applied to the interaction volume during the electron time of flight in order to suppress random coincidences with ions coming from previous synchrotron radiation pulses. The detection of an electron served as a trigger for a $62 \mathrm{~V} / 15 \mathrm{~mm}$ extraction pulse for the ions. Electron time of flight, ion time of flight, and ion impact positions on the anode were recorded in coincidence. The resulting experimental coincidence spectra were corrected afterwards for random coincidences with normalized spectra obtained by applying pulses from a pulse generator instead of electron signals as a trigger for the ion extraction pulse. The homogeneity of the applied electric field has been proven by simulation of the prominent $\mathrm{CO}^{+}, \mathrm{CO}^{2+}$, and $\mathrm{Ar}^{2+}$ peaks which appeared to be in very good agreement with the experimental peak shape. 


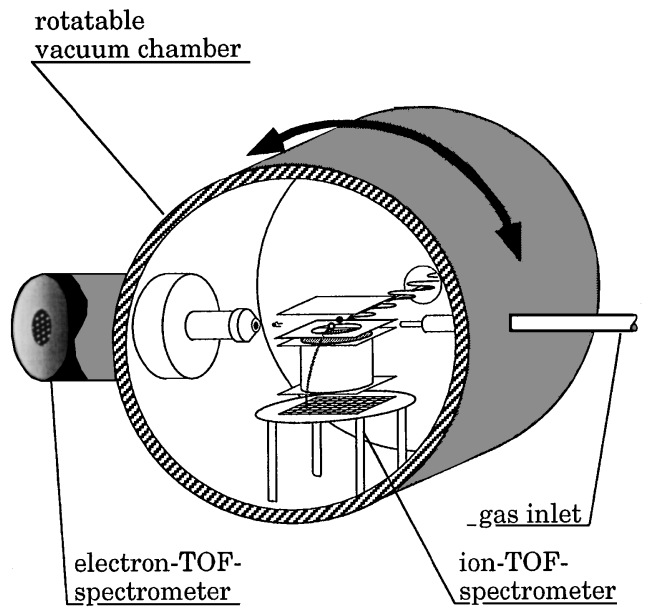

FIG. 1. Scheme of the experimental setup: A rotatable chamber with a time-of-flight electron spectrometer with small acceptance angle and a time-of-flight ion spectrometer with a position-sensitive multihit detection anode for mass and angle resolved photoelectron-photoion coincidence spectroscopy.

Photoionization into nonfragmenting, stable $\mathrm{CO}^{+}$ions is characterized by a sharp peak in the center of the anode whereas ionic fragmentation into $\mathrm{C}^{+}$and $\mathrm{O}^{+}$ ions yields a two-dimensional intensity pattern which reflects the angular distribution of the fragments. Again, simulation of this three-dimensional $\mathrm{CO}^{+}$peak shape provided information on the homogeneity of the electric field. Figure 2 shows one example for each intensity distribution.

The ejection angles of the fragment ions were calculated with a well-calibrated computer simulation using analytical functions, not a finite element method. This is possible because all electric fields in the ion spectrometer are highly homogeneous.

The complete angular distributions of molecular orientations were measured for several fixed directions of the electron detector with respect to the electric vector of the incoming soft $\mathrm{x}$-ray radiation. Normalization and transformation of different molecular-orientation distributions for fixed electron directions lead to electron-emission distributions for fixed molecular orientations. In this

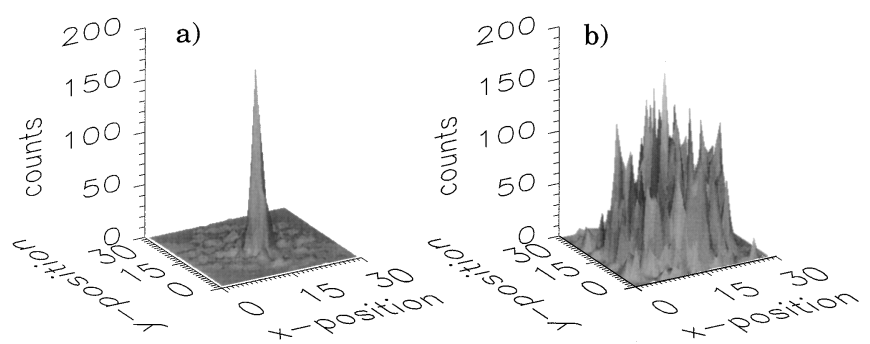

FIG. 2. (a) Intensity distribution of photoion hit positions on the anode following photoionization into stable $\mathrm{CO}^{+}$ions. (b) Two-dimensional hit pattern of $\mathrm{C}^{+}$and $\mathrm{O}^{+}$ions produced in fast ionic fragmentation.
Letter we show the photoemission angular distributions observed for either parallel or perpendicular orientation of the molecular axis with respect to the electric vector of the light. These distributions can be described straightforwardly by

$$
I(\theta)=\sum_{k=0}^{2 l_{\max }} A_{k} P_{k}(\cos \theta)
$$

where $P_{k}$ are Legendre polynomials of $k$ th order, with $l_{\max }$ as maximum angular momentum and $\theta$ the angle between the molecular axis and the electron emission direction [6].

All results shown in this Letter are extracted from the angular distribution of $\mathrm{O}^{+}$ions. All data are averaged over a total kinetic energy of the outgoing $\mathrm{O}^{+}$ion between 1.7 and $4.2 \mathrm{eV}$ and an acceptance angle of $20^{\circ}$. Figure 3 shows a polar plot of the measured angular distribution along with a curve representing a fit to an expansion of Legendre polynomials up to the sixth order, representing a superposition of partial waves up to $l=3$. Figure 3 shows a distinct forward-backward asymmetry, particularly for the case of parallel orientation of the molecular axis with respect to the electric vector. The fit also shows some minor remains of the $f$-wave enhancement in the shape resonance, exhibiting the same strong forward-backward asymmetry. It is interesting to compare these experimental results with the early prediction of Dill, Siegel, and Dehmer [6].

Shigemasa et al. [3] pointed out in their paper that the three-dimensional plots of Dill, Siegel, and Dehmer [6] could not be easily converted into a two-dimensional representation for a certain photon energy. Detailed examination of the calculated data of Ref. [6] and polynomial interpolation of the missing data points, however, shows that it is indeed possible to derive angular distributions for certain photon energies from these figures. Figure 4 shows the corresponding curve for a photon energy of $321 \mathrm{eV}$ in comparison with the experimental data and the curve obtained by a Legendre polynomial fit. The agreement is unexpectedly good, in particular

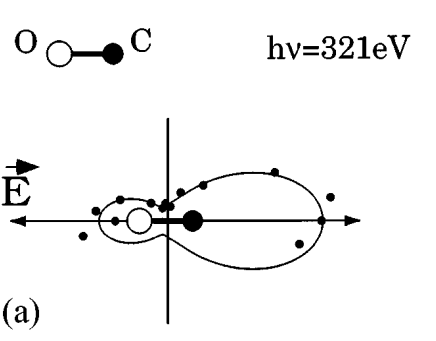

(b)

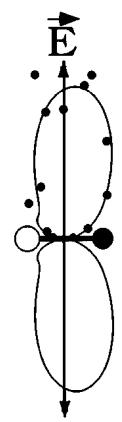

FIG. 3. Polar representation of the measured angular distribution of photoelectrons in coincidence with photoions emitted (a) along and (b) perpendicular to the electric vector. The solid curves represent a fit to an expansion of Legendre polynomials. 

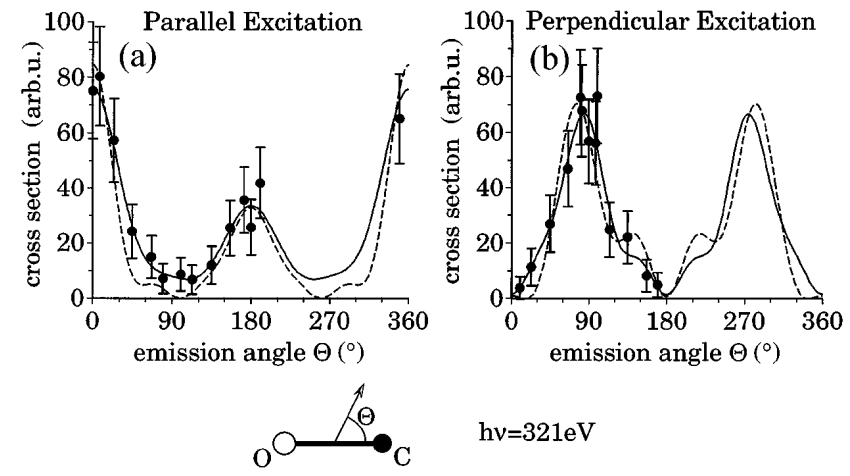

FIG. 4. The data points (filled circles) with error bars and the fitting curves (solid lines) of Fig. 3 in Cartesian coordinates compared with the results of a multiple scattering calculation of Dill, Siegel, and Dehmer [6] (dashed lines).

if one keeps in mind that the MS-X $\alpha$ method seems to overestimate the $f$-wave enhancement in the shape resonance. Our measurements show that this effect still remains for photon energies several electron volts above resonance where the calculated $f$-wave components are overestimated by approximately a factor of 2 . However, the degree of forward-backward asymmetry seems to be nearly perfectly reproduced by the $\mathrm{MS}-\mathrm{X} \alpha$ method. Because this asymmetry as an interference effect is more sensitive to intramolecular scattering than the angular momentum composition, it is not that surprising that the molecular scattering model does work quite well in this respect. The interference between scattered and unscattered photoelectron waves depends on the bond lengths of a diatomic molecule or, more general, on the topology of a molecule. As a result the emission intensities along a certain direction with respect to the molecular axis vary with the photon energy. These oscillations are most pronounced for forward and backward photoelectron emission - a behavior which is, in fact, analog to the photoelectron diffraction in a free molecule. Finally, we compare our results with the mass integrated measurements of Heatherly et al. on $\mathrm{CO}$ at $h \nu=325.8 \mathrm{eV}$ [4]. Figure 5 shows the results of these authors along with our mass averaged results and the corresponding fitting and theoretical MS-X $\alpha$ curves. The agreement is quite good, showing that the reported results obtained by a new method are not affected by systematic deviations as, for example, spatially dependent efficiency problems of the position-sensitive detector.

In summary, we have shown for the first time that photoelectron angular distributions from oriented heteronuclear molecules show large forward-backward asymmetries pointing to intrinsic molecular scattering as their origin. These observations are in excellent agreement
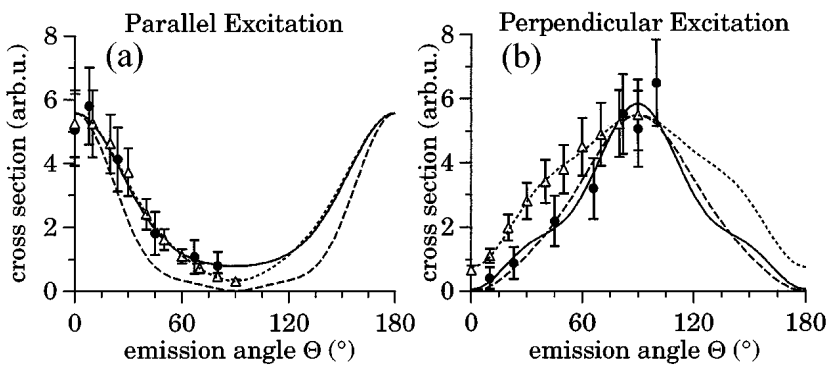

FIG. 5. Mass-averaged results of the present experimental data (filled circles) and MS-X $\alpha$ calculation of Ref. [6] compared with the mass-integrated measurements of Heatherly et al. [4] (open triangles) taken at $h \nu=325.8 \mathrm{eV}$ for (a) parallel and (b) perpendicular excitation. The solid, dashed, and dotted lines represent the corresponding curves of this work, the MS-X $\alpha$ calculation of Dill, Siegel, and Dehmer [6], and the fitting results of Ref. [4], respectively.

with predictions made on this subject twenty years ago by Dill, Siegel, and Dehmer [6] using the MS-X $\alpha$ method. The results may open the field of photoelectron diffraction studies on free molecules as a tool for structural studies of diatomic and more complex molecules.

We thank the staff at HASYLAB for the excellent support, and the Deutsche Forschungsgemeinschaft for funding this project.

[1] J. L. Dehmer and D. Dill, Phys. Rev. Lett. 35, 213 (1975).

[2] J. L. Dehmer and D. Dill, J. Chem. Phys. 65, 5327 (1976).

[3] E. Shigemasa, J. Adachi, M. Oura, and A. Yagishita, Phys. Rev. Lett. 74, 359 (1995).

[4] P. A. Heatherly, J. Adachi, E. Shigemasa, and A. Yagishita, J. Phys. B 28, 2643 (1995).

[5] E. Shigemasa, J. Adachi, M. Oura, N. Watanabe, K. Soejima, and A. Yagishita, in Proceedings of Oji International Seminar on Atomic and Molecular Photoionization, Tsukuba, Japan, 1995, edited by A. Yagishita and T. Sasaki, Frontiers Science Series 18 (Universal Academy Press, Inc., Tokyo, Japan, 1995), p. 69.

[6] D. Dill, J. Siegel, and J.L. Dehmer, J. Chem. Phys. 65, 3158 (1976).

[7] A. de Castro and R. Reininger, Rev. Sci. Instrum. 63, 1317 (1992).

[8] C.U.S. Larsson, A. Beutler, O. Björneholm, F. Federmann, U. Hahn, A. Rieck, S. Verbin, and T. Möller, Nucl. Instrum. Methods Phys. Res., Sect. A 337, 603 (1994).

[9] N. Saito, F. Heiser, O. Hemmers, K. Wieliczek, J. Viefhaus, and U. Becker, Phys. Rev. A 54, 2004 (1996).

[10] R. M. Wood, Q. Zheng, M. A. Mangan, and K. Edwards, Nucl. Instrum. Methods Phys. Res., Sect. B 99, 39 (1995). 